\title{
Non-invasive assessment of Durability of Ablation Lesions with Magnetic Resonance Imaging: are we there yet?
}

\author{
Dhiraj Gupta ${ }^{1}$ \\ ${ }^{1}$ University of Liverpool
}

July 7, 2020

Pulmonary Vein Isolation (PVI) remains the cornerstone for catheter ablation for atrial fibrillation (AF). Achieving durable PVI safely with Radiofrequency Catheter Ablation (RFCA) has proven challenging until recently, even with the use of Contact Force $(\mathrm{CF})$ sensing catheters and electroanatomical mapping ${ }^{1}$. Ablation success rates improve markedly, including in persistent AF, when permanent PVI can be achieved ${ }^{1,2}$, which only underscores the critical role of the Pulmonary Veins (PV) in AF arrhythmogenesis.

Historically, the only way to assess PVI durability has been through invasive electrophysiology study, with all its associated risk, inconvenience, and costs. This price appears particularly galling to pay if the PVs are found to be isolated at repeat study, as is now becoming increasingly common ${ }^{3}$. Multiple randomised studies have failed to show additional benefit from ablating extra-PV structures ${ }^{4,5}$, and the best outcomes following repeat $\mathrm{AF}$ ablation procedures are restricted to those where PV reconnection is identified and treated $^{6}$. As such, there remains a pressing need for a non-invasive tool that can accurately assess PVI durability, and ideally, the size and location of residual gaps. As Magnetic Resonance Imaging (MRI) has increasingly been shown capable of delineating atrial scar, there is much anticipation that it may serve this important purpose ${ }^{7}$.

RFCA and Cryoballoon ablation (CBA) are by far the most common modalities used for PVI, and there is remarkable equivalence in their clinical results ${ }^{8}$. However, the handling of the two technologies in the catheter laboratory is very different, and ultrahigh density mapping has shown important differences in the number and location of chronic gaps between the two ${ }^{9}$. The use of MRI in characterizing these differences has not been well described so far.

In this issue of the journal, Kurose and colleagues present a small but elegant study ${ }^{10}$, in which 30 consecutive patients who underwent PVI (18 with CBA, 12 with RFCA) were assessed by LGE-MRI two months later, where lesion width and visual gap(s) around each vein were assessed. The RF applications were delivered using a CF sensing catheter, with a target lesion size index (LSI) of 5 , and an inter-lesion distance of $<6 \mathrm{~mm}$. They found that the mean lesion width on MRI was significantly wider in the CBA group $(8.1 \pm 2.2 \mathrm{~mm})$ as compared to the RFCA group $(6.3 \pm 2.2 \mathrm{~mm}), \mathrm{p}=0.032$. However, there were more visual gaps seen in the CBA group, especially in the bottom segments of the two inferior veins. In the RFCA group, gaps were seen most often seen in the left posterior segments where the target LSI value could not be achieved because of esopheageal temperature rise. Furthermore, the number of gaps visualised on MRI was linked to freedom from $\mathrm{AF}$ at 12 months; receiver operating characteristic curve analysis suggested a cut off value of less than 5 visual gaps per patient as being predictive of a good outcome.

The authors deserve to be congratulated for their study, which builds on their previous work where LGEMRI was used to compare chronic lesions between CBA and RFCA with non-CF sensing catheters ${ }^{11}$. It is notable that whilst the lesion width in their previous study was also significantly greater in the CBA group than the RFCA group, the mean number of gaps in the RFCA group was higher. This suggests that the 
modern technique of delivering LSI-guided contiguous RFCA lesions has resulted in a material improvement in PVI durability, something that is borne out in clinical studies too ${ }^{3}$.

Some limitations of the work should be mentioned. Patients were not randomised to RFCA or CBA; rather, patients undergoing CBA were pre-selected with those with left common PV or large PVs excluded. The ablation technique used for CBA was unusual in that the use of RFCA was allowed if PVI could not be achieved after a single 3-minute freeze. This low bar for defining CBA failure led to as many as 3 patients out of 25 being excluded from the study. Many readers will feel that the mean procedural times of 129 minutes and fluoroscopy times of 39 minutes for CBA are much longer than what is the norm today. They may also find the RF powers used in this study unusual; only $30 \mathrm{~W}$ was used on the anterior wall, and $20-25 \mathrm{~W}$ on the posterior wall, which was reduced even further if esophageal temperature rise was observed. The field is moving towards using higher power short duration (HPSD) RF applications, and as HPSD lesions have been shown to be wider ${ }^{12}$, it is possible that the gaps on the posterior wall identified in this study may not have been present had HPSD applications been used. Finally, the definition of visual gap on MRI used in this study, a non-LGE site larger than $4 \mathrm{~mm}$, almost certainly overestimated the number of true gaps. For instance, the authors observed at least one visual gap in each of the 16 segments around the PVs in more than $10 \% \mathrm{CB}$ patients; this is at odds with data obtained with ultrahigh density mapping ${ }^{9}$, and also with the good clinical outcomes reported here. Future research should look at correlating these MRI-visualised gaps with actual gaps seen on repeat electrophysiological study, so that the clinical significance of these can be better defined.

What can we take away from this study? Firstly, the use of MRI to assess post-ablation scar is now a reality in many labs, allowing assessment of PVI durability to help decide whether or not to offer a repeat procedure to a patient with AF recurrence. Secondly, the evolution of the RFCA technique to include target lesion indices and inter-lesion distance has made RFCA at least as effective as CBA in achieving durable PVI. Finally, this is an area ripe for further research, and we look forward to similarly valuable contributions from Kurose and colleagues in the future.

\section{References}

1. Das M, Wynn G, Saeed Y, et al. Pulmonary vein re-isolation as a routine strategy regardless of symptoms: The PRESSURE randomized controlled trial. JACC Clin Electrophysiol 2017;3(6):602-61

2. Hussein A, Das M, Riva S, et al. Use of ablation index-guided ablation results in high rates of durable pulmonary vein isolation and freedom from arrhythmia in persistent atrial fibrillation patients. Circ Arrhythm Electrophysiol 2018;11: e006576.

3. De Pooter J, Strisciuglio T, El Haddad M, et al. Pulmonary Vein Reconnection No Longer Occurs in the Majority of Patients After a Single Pulmonary Vein Isolation Procedure. JACC Clin Electrophysiol 2019;5(3):295-305

4. Verma A, Jiang C, Betts TR, et al. Approaches to Catheter Ablation for Persistent Atrial Fibrillation. N Engl J Med 2015 May 7;372(19):1812-22

5. Wynn GJ, Panniker S, Morgan M, et al. Biatrial linear ablation in sustained nonpermanent AF: results of the substrate modification with ablation and antiarrhythmic drugs in nonpermanent Atrial Fibrillation (SMAN-PAF) trial. Heart Rhythm 2016;13(2):399-406

6. Kim T-H, Park J, Uhm J-S, et al. Pulmonary vein reconnection predicts good clinical outcome after second catheter ablation for atrial fibrillation. Europace 2017;19(6):961-967

7. Ranjan R, Kato R, Zviman MM, et al. Gaps in the ablation line as a potential cause of recovery from electrical isolation and their visualization using MRI. Circ Arrhythm Electrophysiol. 2011;4(3):279-286.

8. Andrade J, Champagne J, Dubuc M, et al. Cryoballoon or radiofrequency ablation for atrial fibrillation assessed by continuous monitoring: a randomized clinical trial. Circulation 2019;140:1779-1788

9. Gunawardene MA, Eickholt C, Akbulak RÖ, Jularic M, Klatt N, Hartmann J, Schlüter M, Meyer C, Willems S, Schaeffer B. Ultra-high-density mapping of conduction gaps and atrial tachycardias: distinctive patterns following pulmonary vein isolation with cryoballoon or contact-force-guided radiofrequency current. J Cardiovasc Electrophysiol 2020;31(5):1051-1061 
10. Kurose J, Kiuchi K, Fukuzawa K, et al. Lesion characteristics between cryoballoon ablation and radiofrequency ablation with a contact-force sensing catheter: late-gadolinium enhancement magnetic resonance imaging assessment. J Cardiovasc Electrophysiol 2020 (in press)

11. Kurose J, Kiuchi K, Fukuzawa K, et al. The lesion characteristics assessed by LGE-MRI after the cryoballoon ablation and conventional radiofrequency ablation. J Arrhythm. 2018;34(2):158-166.

12. Leshem E, Zilberman I, Tschabrunn CM, et al. High-power and short-duration ablation for pulmonary vein isolation: biophysical characterization. JACC Clin Electrophysiol 2018 Apr;4(4):467-479 